\title{
Transfusion Medicine Practice in a Pandemic; Use of Convalescent Plasma in the Management of COVID-19
}

\section{Sarah Tehseen}

Saskatchewan Health Authority, Canada CA.

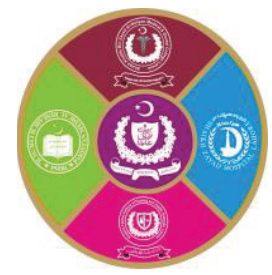

C OVID-19 pandemic has now infected over 1 million people and caused 62,000 deaths around the world as of April $5^{\text {th }}, 2020 .{ }^{1}$ It is caused by a virus of Coronaviridae family now named SARS-CoV (2) which leads to severe viral pneumonia and acute respiratory distress syndrome (ARDS). ${ }^{2}$ The number of cases continue to rise globally with a growing increase in mortality. At this time, no specific treatment is found to be curative for COVID-19 however multiple experimental treatments are being explored. ${ }^{3}$

Current therapy for COVID-19 is mainly supportive with a focus on prevention by reducing and containing the spread of the virus. ${ }^{3}$ The experimental therapies being investigated include antivirals like lopinavir-ritonavir, interferon-1 $\beta$, the RNA polymerase inhibitor Remdesivir and chloroquine. ${ }^{4}$

The use of plasma from convalescent COVID-19 patients is an attractive option since it provides passive immunity by neutralizing antibodies and has precedence in the management of viral pneumonia caused by other viruses of Coronoviridae family as well. Though the quality of evidence of available literature is moderate to low, the studies showed a consistent reduction in mortality among patients with influenza or SARS-CoV (1). ${ }^{5}$ Blood supplier systems in Canada and the US are currently working towards developing clinical trials to investigate the effect of convalescent plasma on morbidity and mortality from COVID-19. ${ }^{6,7}$ FDA has approved its use for critically ill patients following expedited approval on a case by case basis. ${ }^{12}$

\footnotetext{
The Pathophysiological basis for use of Convalescent Plasma:

Both SARS-CoV(1) and the novel SARS-CoV-2 are highly pathogenic Coroviruses with a strong affinity for lungs. These viruses enter the target cells by (S) spike glycoproteins through the ACE-2 receptors. It is postulated that the neutralizing antibodies against these viruses inhibit the S spike mediated entry into target cells. ${ }^{8}$ Multiple animal models have also shown that these antibodies prevent re-infection and viral replication both SARS CoV (1) and CoV (2) viruses. ${ }^{9,10}$
}

The source of these neutralizing antibodies can be human convalescent plasma or specific preparations in animal hosts. The human convalescent plasma can then be fractionated and treated to form hyperimmune globulin. Hyper-immune globulin and animal-based antibody sources both require multiple processing steps which will be ultimately beneficial but cannot provide a rapid source of antibodies for the current epidemic. Hence, currently, straight administration of convalescent COVID-19 plasma seems to be the most rational step in the management of critically ill patients. ${ }^{13,14}$

As mentioned above, plasma from convalescent patients will act through neutralizing antibodies that prevent viral entrance into target cells and potential replication. Other mechanisms such as antibodymediated cellular cytotoxicity and phagocytosis may also be possible. ${ }^{14}$

A recent report of five critically ill individuals with ARDS and SARS-CoV (2) infection indicated resolution of ARDS in 2 patients and an overall improvement in respiratory status with no mortality in all 5 patients. All patients demonstrated a significant reduction in viral load. These patients received plasma from five donors who had recovered from SARS CoV (2) infection with high titers of neutralizing antibody. ${ }^{11}$ This case series in human subjects suggests a beneficial effect of the use of convalescent plasma, however, it is not possible to determine the extent of benefit from convalescent plasma as these patients were receiving multiple therapies at the same time including antivirals and anti-inflammatory agents.

Potential Complications of Plasma
Administration:
Human plasma administration is not risk-free and
can lead to mild as well as severe complications
such as transfusion reaction associated lung injury
(TRALI) and anaphylaxis. However, most studies
do not indicate any significant side effects of plasma
transfusion. ${ }^{5}$ There were two reported cases of
possible TRALI after the use of convalescent
plasma for an Ebola and a middle eastern respiratory
syndrome (MERS) patient. ${ }^{15,16}$
Another consideration is the pro-inflammatory
characteristics of plasma and antibodies which may


exacerbate the ARDS contracted by COVID-19 patients. Although the previous reports on the use of convalescent plasma as well as the current case series of 5 patients report no such adverse effects, this risk should be contemplated when deciding on plasma administration.

\section{Dose and Timing of Plasma Administration:}

There is no recommended dose of plasma for COVID-19 patients. The patients treated during the SARS-CoV2 epidemic received two consecutive transfusions of 200-250 ml of ABO compatible plasma collected from donors on the same day ${ }^{11}$ Patients with Ebola virus were also treated with a similar dose. ${ }^{17}$ Clinical trials being designed to study the impact of convalescent plasma are using 2 doses of 200-250ml of plasma over 1-2 days. ${ }^{23}$

Data obtained from the use of convalescent plasma for viral illnesses has shown its maximum benefit when administered early during disease. It can be hypothesized that the rapid reduction of high viral inoculum with neutralizing antibodies leads to the blunting of pro-inflammatory response and ARDS compared to late administration when the inflammatory response has been established. ${ }^{18}$ In the reported case series on the use of human plasma for COVID-19 patients, it was given between days 1022 of hospital admission. ${ }^{11}$ Among patients infected with SARS CoV-1, the plasma was administered on day 14 of admission according to observational studies $^{18}$ The use of convalescent plasma is only investigated in patients with severe respiratory illness needing significant support. ${ }^{7,11}$

\section{Donor Perspectives:}

Donors should be considered for plasma collection after complete resolution of symptoms for at least 28 days before donation or resolution of symptoms for 14 days with a negative test for SARS-CoV2. ${ }^{7}$ They can be contacted through the hospital registries after discharge or through widespread educational and marketing campaigns catered to both public and healthcare providers. Screening the population for titers of $\operatorname{IgG}$ antibodies against SARS-CoV2 as potentials for plasma donation is another option but has significant budgetary constraints. $^{21}$

These donors should obey the local guidelines for donor eligibility. At the very least infectious disease testing for transfusion-transmitted disease must be performed before accepting the plasma donation. It is recommended that plasma should be collected from men and women with no prior history of pregnancy to mitigate the risk of TRALI. ${ }^{7}$
The titers of neutralizing antibodies in the plasma of convalescent SARS-CoV(1) patients reached a peak at 4 months and continued to decline afterward. ${ }^{19}$ The patterns of antibody rise and decline in SARSCoV (2) convalescent patients are still undetermined but it is likely beneficial to consider plasma donation in the first 4 months after diagnosis of COVID-19 for maximal benefit. It is also interesting that individuals with severe illness due to SARSCoV1 were more likely to have high titers of neutralizing antibodies in their plasma. Whether this holds for SARS-CoV2 as well needs to be elucidated. ${ }^{20}$

Ideally, the collected plasma should be tested for titers of neutralizing antibodies against SARSCoV2. Current clinical trials in the US are using a minimum titer of 1:64 as a criterion for plasma collection based on prior research on SARS-CoV1. ${ }^{22}$ However, the determination of antibody titers may prove to be difficult in resource-limited settings. At a minimum, potential donors should be tested for the presence of neutralizing antibodies using ELISA or similar techniques. ${ }^{21}$

\section{Plasma Collection and Storage:}

Plasma collection can be undertaken using pheresis machines or following a whole blood donation. One benefit of pheresis is that it will allow the donor to donate plasma every 2 weeks whereas whole blood donations can only be performed every 12 weeks. Whole blood donation and subsequent separation and storage of plasma may be more applicable to resource-limited settings and should be considered. After collection from convalescent donors, plasma can be donated immediately or frozen at -18 degrees Celsius for up to 12 months for later use or pooled to form hyperimmune globulin. Pathogen reduction technique for viral inactivation of plasma, if available, should be performed before plasma administration. ${ }^{17}$

\section{CONCLUSION}

There are no proven therapeutic modalities for the cure of COVID-19. While significant research is underway to develop medical therapies and vaccines, none are available in a time-sensitive fashion. The use of convalescent plasma as a source of passive immunity against the virus is a feasible and timely solution for critically ill patients. This option can be utilized in resource-limited settings as well with appropriate modifications to plasma collection, donor testing, and administration based on locally developed protocols. As plasma administration has some significant risks, its use must be carefully weighed against the benefits when considering 
treatment. Ongoing clinical trials will help determine the optimal dose, timing and the patient cohort most likely to benefit from this treatment modality.

\section{REFERENCES}

1. https://www.who.int/docs/default-source/ coronaviruse/situation-reports/20200405-sitrep76-covid-19.pdf?.sfvrsn=6ecf0977 4

2. Lu, Roujian, et al. "Genomic characterization and epidemiology of 2019 novel corona virus: implications for virus origins and receptor binding." The Lancet 395.10224. 2020;565-574.

3. Fauci, Anthony S., H. Clifford Lane, and Robert R. Redfield. "COVID-19 - navigating the uncharted." 2020; 1268-1269.

4. World Health Organization. WHO R\&D Blueprint: informal consultation on prioritization of candidate therapeutic agents for use in novel corona virus 2019 infection, Geneva, Switzerland, 24 January 2020. No. $\mathrm{WHO} / \mathrm{HEO} / \mathrm{R} \& \mathrm{D}$ Blueprint $(\mathrm{nCoV}) / 2020.1$. World Health Organization, 2020.

5. Mair-Jenkins, John, et al. "The effectiveness of convalescent plasma and hyperimmune immunoglobulin for the treatment of severe acute respiratory infections of viral etiology: a systematic review and exploratory metaanalysis." The Journal of infectious diseases 211.1. 2015; 80-90.

6. Levy, Prokopchuk-Gauc et al. NEBMC statement on convalescent plasma and IVIG for treatment of COVID-19 infection.March31 $1^{\text {st }}, 2020$

7. https://www.fda.gov/vaccines-blood-biologics/ investigational-new-drug-ind-or-deviceexemption-ide-process-cber/investigationalcovid-19-convalescent-plasma-emergency-inds

8. Walls, Alexandra C., et al. "Structure, function, and antigenicity of the SARS-CoV-2 spike glycoprotein." Cell (2020).

9. Subbarao, Kanta, et al. "Prior infection and passive transfer of neutralizing antibody prevent replication of severe acute respiratory syndrome corona virus in the respiratory tract of mice." Journal of virology 78.7. 2004; 3572-7.

10. Bao L, Deng W et al. Re-infection could not occur in SARS-CoV (2) infected rhesus macaques. Boprxiv. 20200313 (not yet peerreviewed)

11. Shen, Chenguang, et al. "Treatment of 5 Critically Ill Patients with COVID-19 with Convalescent Plasma." JAMA. 2020.
12. Tanne, Janice Hopkins. "COVID-19: FDA approves use of convalescent plasma to treat critically ill patients." 2020.

13. Beigel, John H, et al. "Safety and tolerability of a novel, polyclonal human anti-MERS corona virus antibody produced from transchromosomic cattle: a phase 1 randomized, double-blind, single-dose-escalation study." The Lancet Infectious Diseases 18.4. 2018; 410-18.

14. Casadevall, Arturo, and Liise-anne Pirofski. "The convalescent sera option for containing COVID-19." The Journal of clinical investigation 130.4 (2020).

15. Mora-Rillo, Marta, et al. "Acute respiratory distress syndrome after convalescent plasma use: treatment of a patient with Ebola virus disease contracted in Madrid, Spain." The Lancet Respiratory Medicine 3.7. 2015; 554-62.

16. Chun, Sejong, et al. "Possible transfusionrelated acute lung injury following convalescent plasma transfusion in a patient with the Middle East respiratory syndrome." Annals of laboratory medicine 36.4. 2016; 393-5.

17. Tiberghien, Pierre, et al. "Collecting and evaluating convalescent plasma for COVID-19 treatment: why and how." Vox Sanguinis. 2020.

18. Momattin, Hisham, et al. "Therapeutic options for Middle East respiratory syndrome coronavirus (MERS-CoV)-possible lessons from a systematic review of SARS-CoV therapy." International Journal of Infectious Diseases 17.10 .2013; 792-8.

19. Cao, Wu-Chun, et al. "Disappearance of antibodies to SARS-associated corona virus after recovery." New England Journal of Medicine 357.11. 2007; 1162-3.

20. Hung, Ivan FN, et al. "Effect of clinical and virological parameters on the level of neutralizing antibody against pandemic influenza A virus H1N1 2009." Clinical Infectious Diseases 51.3. 2010; 274-9.

21. Focosi, Daniele, et al. "CONVALESCENT BLOOD PRODUCT THERAPIES FOR COVID-19: A SYSTEMATIC REVIEW."2020.

22. https://clinicaltrials.gov/ct2/show/study/NCT04 325672

23. https:/clinicaltrials.gov/ct2/show/NCT04332835

The Authors:

Dr. Sarah Tehseen

Saskatchewan Health Authority, Canada CA.

Email: sarah.tehseen@gmail.com 